\title{
A 30 Year-Old Man Infectious Endocarditis and Cerebrovascular AcCident
}

Neerav G. Sheth, MD, Joseph DeSimone, MD

\section{Case Report}

A 30 year old male with a remote history of intravenous drug abuse (IVDA) and Hepatitis C was admitted in July 2008 with changes in mental status and a new right sided paresis. According to the family, the patient was in his usual state of health until approximately 2-3 weeks prior to admission when he developed headaches, generalized malaise, and fever to 101 degrees Fahrenheit after swimming in a lake. He was seen in an outside hospital emergency department, diagnosed with otitis media and subsequently discharged on prednisone and ciprofloxacin. The morning of admission, the patient was found naked, nonverbal, confused and was subsequently taken again to an outside hospital emergency department where he was found to have right sided weakness, diagnosed with a new cerebrovascular accident (CVA) and transferred to TJUH for further management.

On admission, the patient was found to have persistent right sided hemiparesis, a new loud systolic murmur best heard at the left lower sternal border and petechiae on his calves. Initial labs revealed a leukocytosis with left shift. His CT scan showed an acute left MCA territory infarction (Figure 1). A follow up MRI revealed additional punctuate foci of restricted diffusion in the right parietal and left occipital lobe (Figure 2), suggestive

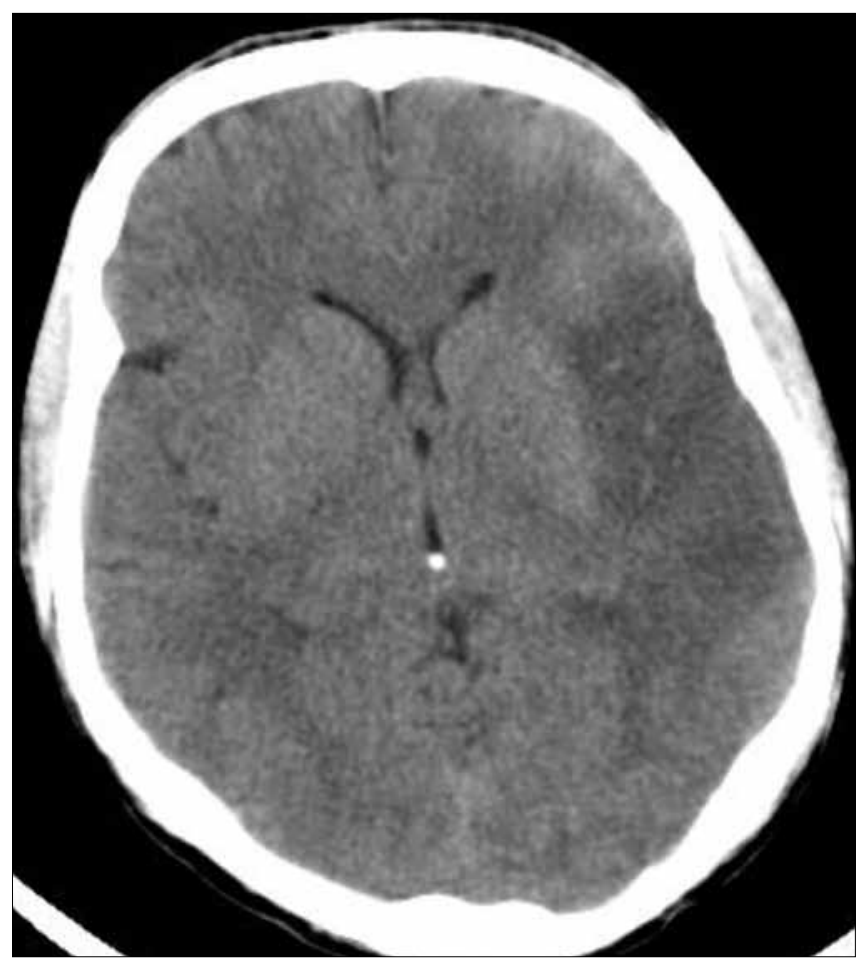

Figure 1. CT scan showing Hypoattenuation in Left Frontal and Temporal lobes compatible with Acute Left MCA Territory Infarct.

of embolic source of the patient's CVA. The patient had a transthoracic echocardiogram which showed a myxomatous appearing mitral valve with an echodensity near the base of the posterior mitral valve with vegetations (Figure 3). A transesophageal echocardiogram revealed a mobile $0.5 \mathrm{~cm} \times 0.5$ $\mathrm{cm}$ echodensity attached to the posterior mitral valve leaflet as well as a second $0.9 \mathrm{~cm} \times 0.5 \mathrm{~cm}$ echodensity attached to the anterior mitral valve leaflet (Figure 4). Of note, the patient's TEE bubble study revealed patent foramen ovale (PFO), which is postulated to have contributed to the presence left sided infectious endocarditis in a patient with IVDA.

Given the patient's history of IVDA, he was started on vancomycin for Staphylococcus aureus via a peripherally inserted central catheter. A few days later, the organism was found to be sensitive to methicillin and antibiotics were adjusted apropriately.

\section{Discussion}

Infectious endocarditis (IE) has been described in the medical literature for over 100 years. Prior to antibiotics, the condition was uniformly fatal, with a majority of cases diagnosed at autopsy. It wasn't until the development of echocardiography that there was a significant reduction in the morbidity and mortality and mortality of IE.

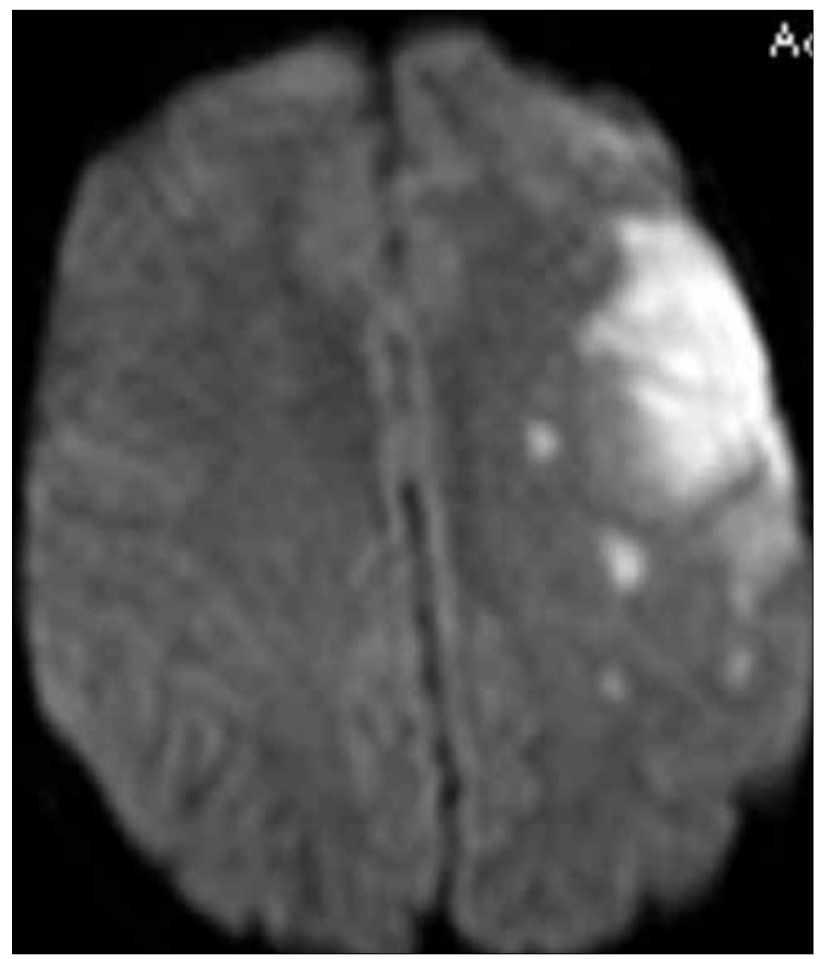

Figure 2. MRI showing acute infarct involving left frontal and parietal lobes in left MCA distribution with punctate foci of diffusion restriction consistent with embolic phenomena. 


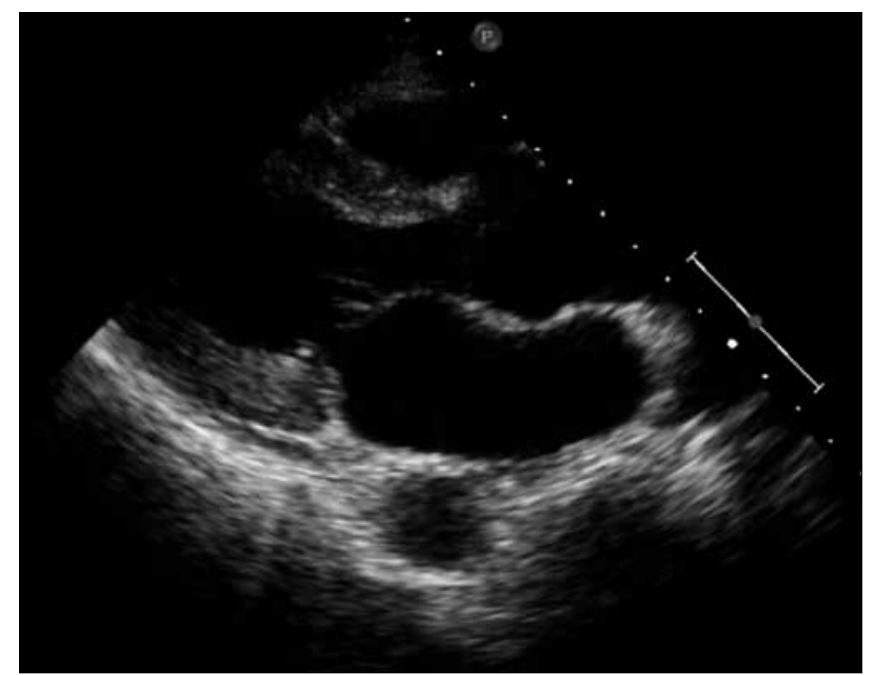

Figure 3. TTE showing myxomatous appearance of mitral valve with vegetation

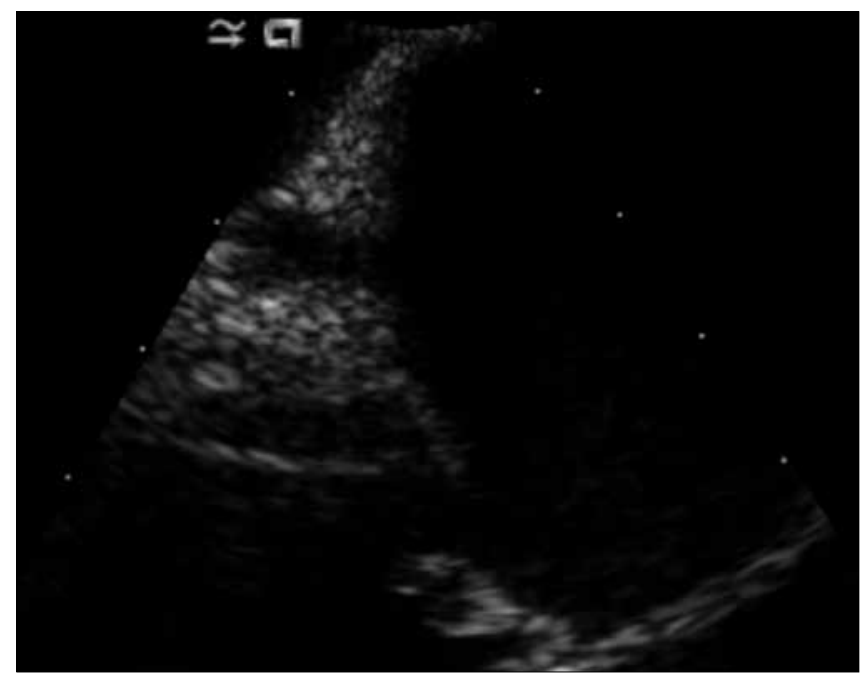

Figure 4. TEE showing large vegetation on mitral valve.

Presently, there are 10-15,000 new cases of IE diagnosed in the United States each year ${ }^{1}$ with estimated national incidences ranging from 1.4 to 6.2 per $100,000 .^{2}$ Moreover, epidemiology reports from the early 1990's, report incidences as high as 11 per 100,000 in the Philadelphia area. ${ }^{1}$ In particular, IE has become more a disease of the elderly as over half of all new cases occur in those over the age of $60 .^{3}$

This older demographic, in addition to previously noted conditions of intravenous drug abuse, structural heart disease, and recurrent bacteremia may have additional risk factors that predispose them to develop IE. These include patients on hemodialysis, with indwelling catheters, severe native and/ or artificial valve disease, diabetes, intravenous drug abuse, and HIV infection. These additional factors, coupled with a significant decline in rheumatic heart disease as a risk factor in younger patients has resulted in an increase in the median age of IE patients over the past 40 years. ${ }^{3}$

Independent of age or risk factors, the most common offending organisms remain the same, with Staphylococcus aureus and Streptococcus spp. (including viridians, bovis, and enterococcus) being the most common. They have been associated with both native and diseased valve endocarditis. Gram negative bacteria including Haemophilus species (Haemophilus parainfluenzae, Haemophilus aphrophilus, and Haemophilus paraphrophilus), Actinobacillus actinomycetemcomitans, Cardiobacterium hominis, Eikenella corrodens, and Kingella species (HACEK organisms) have also been described as causes of infectious endocarditis, particularly in non-IVDA patients. These organisms have an increased affinity for adherence to native valve tissue. However, they are much less common in comparison to S. aureus and Streptococcus spp and are responsible for only 5-10\% of non-IVDU native valve IE cases. ${ }^{4}$

Those patients that develop IE, with or without prophylaxis, may present with a wide variety of clinical features. Some may present with classic signs and symptoms of low grade fevers, a new murmur, petechiae, Roth spots, Janeway lesions, and Osler's nodes. The last three findings are highly specific and pathognomonic for IE, but have a low rate of occurrence. Often, some of the features may not be visible at the time of presentation. Occasionally, some patients, including the one presented, will present with symptoms of embolic phenomena.

The diagnosis of IE primarily includes clinical and echocardiographic criteria, divided into major and minor criteria (Table 1). Recently, the Duke Criteria were modified to include the echocardiographic criteria, as well as to distinguish between definite, probable, and rejected categories (Table 2).

Treatment of IE is directed towards the causative organism as well as the degree of valvular dysfunction. Staphylococcal and Streptococcal species are usually responsive to intravenous vancomycin, pencillins or third generation cephalosporins. If

\begin{tabular}{|c|c|}
\hline Major Criteria & Minor Criteria \\
\hline $\begin{array}{l}\text { 1. Blood cultures positive for } \\
\text { typical organisms } \\
\text { 2. positive cultures } 12 \text { hours } \\
\text { apart } \\
\text { 3. } 3 \text { of } 3 \text { positive from } \\
\text { different sites } \\
\text { 4. Majority of }>3 \text { cultures } \\
\text { positive } \\
\text { 5. Echocardiogram positive } \\
\text { for IE }\end{array}$ & $\begin{array}{l}\text { 1. Predisposing heart lesions } \\
\text { 2. IVDA } \\
\text { 3. Fever } \\
\text { 4. Vascular lesions: } \\
\text { 5. Janeway lesions, emboli, } \\
\text { septic infarcts, hemorrhages } \\
\text { 6. Immunologic phenomena } \\
\text { Glomerulonephritis, } \\
\text { Osler's nodes, Roth spots, } \\
\text { Rheumatoid Factor } \\
\text { 7. Microbiologic evidence not } \\
\text { meeting major criteria }\end{array}$ \\
\hline
\end{tabular}




\begin{tabular}{l} 
Table 2. Diagnosis of IE based on modified Duke Criteria \\
in Table 1 [Li, JS, Sexton, DJ, Mick, N, et al. Clin Infect \\
Dis 2000; 30:633] \\
\hline Definite Diagnosis of IE \\
\hline 2 Major OR \\
\hline 1 Major and 3 Minor OR \\
\hline 5 Minor \\
\hline Possible Diagnosis of IE \\
\hline Major and 1 Minor OR \\
\hline 3 Minor \\
\hline Rejected Diagnosis of IE \\
\hline Firm alternate diagnosis other than IE \\
\hline Resolution of symptoms with 4 days of Antibiotics \\
\hline No pathologic evidence of IE \\
\hline No echocardiographic evidence of IE \\
\hline
\end{tabular}

there is significant valvular dysfunction, cardiothoracic surgery consultation is warranted for valve repair and/or replacement. ${ }^{5}$

Since there is a significant morbidity and mortality associated with IE, focus has shifted towards prevention in those patients at increased risk. However, as previously mentioned, the group of patients "at risk" can encompass a wide array of individuals. As a result of this broadening category of "at risk" patients, it was necessary to identify those at high enough risk to warrant prophylaxis with antibiotics. Recently, the American College of Cardiology and the American Heart Association in conjunction with the Infectious Disease Society of America have reviewed the data on prevention of IE and have subsequently revised the guidelines regarding antibiotic prophylaxis (Table 3 ). These guidelines reflect significant changes from previously published ones and are based on the evidence for, or lack thereof, the efficacy of prophylaxis in patients identified as high risk for IE. ${ }^{6}$

Amoxicillin is the antibiotic of choice for the majority of patients requiring prophylaxis and is given in a dose of 2 grams orally approximately 30 to 60 minutes prior to the procedure. Patients with a documented allergy to penicillins may be given any of the following 30 to 60 minutes prior to the procedure: 2 grams of cephalexin, 500 milligrams of either azithromycin or clarithromycin, or 600 milligrams of clindamycin. ${ }^{6}$

In conclusion, IE is a real but preventable entity once the high risk patients have been identified. A review of recent data has greatly narrowed the number of patients considered "high risk" and has simultaneously limited the instances in which antibiotic prophylaxis is necessary. However, narrowing the spectrum of patients in which prophylaxis does not preclude the necessity of a pre-emptive approach in preventing infectious endocarditis and its sequelae.

\section{References}

1. Bayer, AS. Infective Endocarditis. Clin Infect Dis 1993; 17:313.

2. Tleyjeh, IM, Abdel-Latif, A, Rahbi, H, et al. A Systematic review of population based studies of infective endocarditis. Chest 2007; 132:1025.

3. Hill, EE, Herijgers, $P$, Claus, $P$, et al. Infective endocarditis: changing epidemiology and predictors of 6-month mortality: a prospective cohort study. Eur Heart J 2007; 28:196

Table 3. Updated ACC/AHA/ID Society recommendations for prophylaxis against IE6

\begin{tabular}{|c|c|}
\hline 1. & $\begin{array}{l}\text { Ithough IE is a serious condition, the efficacy of antimicrobial prophylaxis is uncertain in all but a few select instances. Theref } \\
\text { eere are no longer any Class I recommendations for IE prophylaxis in patients with valvular heart disease. }\end{array}$ \\
\hline 2. & $\begin{array}{l}\text { Antibiotic prophylaxis during dental procedures would prevent only a small proportion of cases of IE, even if it were } 100 \% \\
\text { effective. }\end{array}$ \\
\hline 3. & $\begin{array}{l}\text { IE prophylaxis during dental procedures is appropriate only in patients with underlying cardiac conditions associated with the } \\
\text { highest risk for adverse outcomes. These conditions include: } \\
\text { a. prosthetic valves or material } \\
\text { b. Prior IE } \\
\text { c. Unrepaired cyanotic congenital heart disease (CHD) including shunts and conduits } \\
\text { d. Complete CHD repair within previous } 6 \text { months } \\
\text { e. Complete CHD repair with residual defects } \\
\text { f. Valve regurgitation secondary to structural abnormalities in cardiac transplant recipients }\end{array}$ \\
\hline 4. & $\begin{array}{l}\text { In such high-risk patients, prophylaxis is appropriate for all dental procedures involving manipulation of gingival tissue or the } \\
\text { periapical region of teeth, or perforation of oral mucosa (Class Ila). }\end{array}$ \\
\hline 5. & Increased lifetime risk for IE alone is not an indication for antibiotic prophylaxis \\
\hline 6. & $\begin{array}{l}\text { Antibiotic IE prophylaxis is no longer indicated in patients with aortic stenosis, mitral stenosis, or symptomatic or asymptomatic } \\
\text { mitral valve prolapse. }\end{array}$ \\
\hline 7. & Antibiotic IE prophylaxis is no longer indicated in adolescents and young adults with native heart valve disease. \\
\hline 8. & $\begin{array}{l}\text { Genitourinary and gastrointestinal tract procedures (transesophageal echocardiography, esophagogastroduodeno } \\
\text { colonoscopy, etc.) do not warrant IE prophylaxis unless active infection is present. }\end{array}$ \\
\hline
\end{tabular}


4. Feder HM, Roberts JC, Salazar JC, Leopold HB, Toro-Salazar O. HACEK endocarditis in infants and children: two cases and a literature review. Pediatr. Infect. Dis. J. 200322 (6): 557-62.

5. Larry M. Baddour, Walter R. Wilson, Arnold S. Bayer, Vance G. Fowler, Jr, Ann F. Bolger, Matthew E. Levison, Patricia Ferrieri, Michael A. Gerber, Lloyd Y. Tani, Michael H. Gewitz, David C. Tong, James M. Steckelberg, Robert S. Baltimore, Stanford T. Shulman, Jane C. Burns, Donald A. Falace, Jane W. Newburger, Thomas J. Pallasch, Masato Takahashi, and Kathryn A. Taubert. Infective Endocarditis: Diagnosis, Antimicrobial Therapy, and Management of Complications: A Statement for Healthcare Professionals From the Committee on Rheumatic Fever, Endocarditis, and Kawasaki Disease, Council on Cardiovascular Disease in the Young, and the Councils on Clinical Cardiology, Stroke, and Cardiovascular Surgery and Anesthesia, American Heart Association-Executive Summary: Endorsed by the Infectious Diseases Society of America. Circulation 111: 3167-3184.
6. Walter Wilson, Kathryn A. Taubert, Michael Gewitz, Peter B. Lockhart, Larry M. Baddour, Matthew Levison, Ann Bolger, Christopher H. Cabell, Masato Takahashi, Robert S. Baltimore, Jane W. Newburger, Brian L. Strom, Lloyd Y. Tani, Michael Gerber, Robert O. Bonow, Thomas Pallasch, Stanford T. Shulman, Anne H. Rowley, Jane C. Burns, Patricia Ferrieri, Timothy Gardner, David Goff, David T. Durack, and The Council on Scientific Affairs of the American Dental Association has approved the guideline as it relates to dentistry. In addition, this guideline has been endorsed by the American Academy of Pediatrics, Infectious Diseases Society of America. Prevention of Infective Endocarditis: Guidelines From the American Heart Association: A Guideline From the American Heart Association Rheumatic Fever, Endocarditis, and Kawasaki Disease Committee, Council on Cardiovascular Disease in the Young, and the Council on Clinical Cardiology, Council on Cardiovascular Surgery and Anesthesia, and the Quality of Care and Outcomes Research Interdisciplinary Working Group. Circulation 116: 1736-1754.

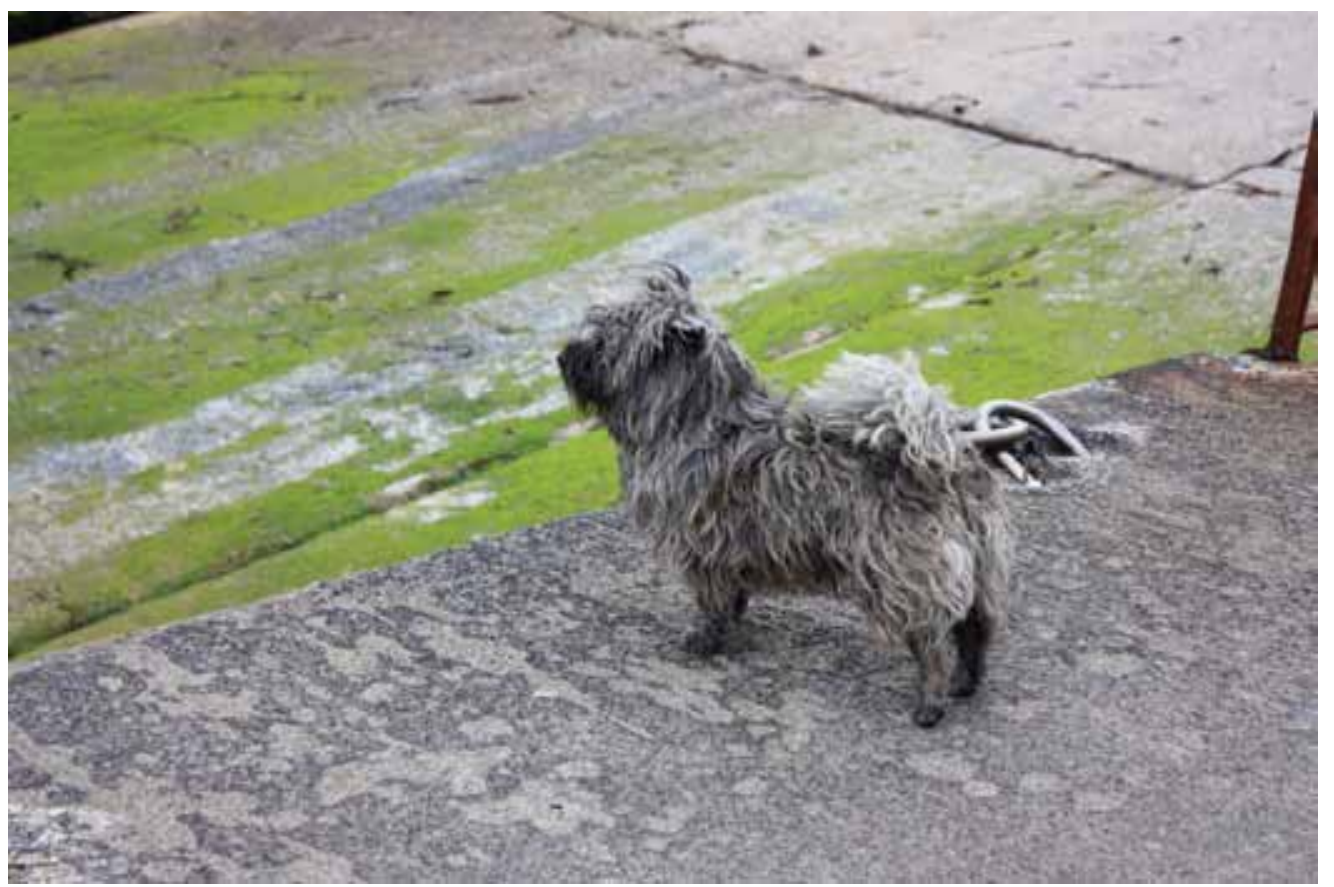

